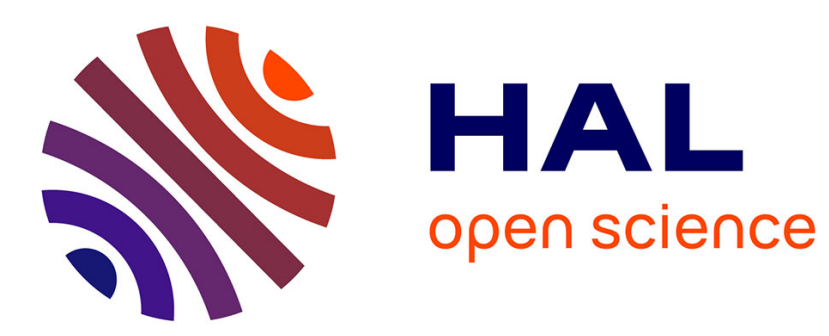

\title{
On the upper critical dimension and the critical exponents of the localization transition
}

\author{
H. Kunz, B. Souillard
}

\section{To cite this version:}

H. Kunz, B. Souillard. On the upper critical dimension and the critical exponents of the localization transition. Journal de Physique Lettres, 1983, 44 (13), pp.503-506. 10.1051/jphyslet:019830044013050300 . jpa-00232223

\section{HAL Id: jpa-00232223 https://hal.science/jpa-00232223}

Submitted on 1 Jan 1983

HAL is a multi-disciplinary open access archive for the deposit and dissemination of scientific research documents, whether they are published or not. The documents may come from teaching and research institutions in France or abroad, or from public or private research centers.
L'archive ouverte pluridisciplinaire HAL, est destinée au dépôt et à la diffusion de documents scientifiques de niveau recherche, publiés ou non, émanant des établissements d'enseignement et de recherche français ou étrangers, des laboratoires publics ou privés. 
Classification

Physics Abstracts

$71.30-71.50$

\title{
On the upper critical dimension and the critical exponents of the localization transition
}

\author{
H. Kunz (*) \\ Institut de Physique Théorique, EPFL, Lausanne, 1006 Suisse
}

and B. Souillard

Centre de Physique Théorique ( ${ }^{* *}$ ), Ecole Polytechnique, F-91128 Palaiseau Cedex, France

(Reçu le 24 mars 1983, accepté le 11 mai 1983).

\begin{abstract}
Résumé. - Nous présentons un ensemble convergent d'arguments conduisant à une dimension critique supérieure $d_{c}=4$ pour la transition de localisation. Ils suggèrent aussi que plusieurs exposants critiques seraient exactement connus, en particulier pour des systèmes tri-dimensionnels, à savoir $v=s .=p=1$.

Abstract. - We present a set of convergent arguments leading to an upper critical dimension $d_{\mathrm{c}}=4$ for the localization transition. They suggest also that several critical exponents would be exactly known, in particular for three-dimensional systems, namely $v=s=p=1$.
\end{abstract}

The concept of an upper critical dimension, above which the critical behaviour of a system becomes simple, i.e. is described by mean field type theories, has played a prominent role in recent theories of critical phenomena. Its value can be 3 (tricritical points), 4 (usual critical points), 6 (percolation)... For the Anderson localization transition, this question remains very much open. One of the main reasons for this situation is that there does not exist yet a simple mean field theory of localization, analogous to the Weiss theory of ferromagnetism. The natural candidate for a simple mean field type theory of localization is Anderson's model on the Bethe lattice, which is thought to represent a space of infinite dimensionality. This model was first analysed by Abou-Chacra, Anderson and Thouless [1] : they showed the existence, in the energydisorder phase-diagram, of a domain of stability of localized states, and a Monte-Carlo simulation gave some information on the behaviour of the system in the region where the non-localized regime is expected, but they did not analyse the neighbourhood of the mobility edge.

The Bethe lattice model is still far from being completely solved and many questions remain unanswered; however we have recently rigorously proven [2] the existence of both localized

$\left({ }^{*}\right)$ Supported by the Fonds National Suisse.

(**) Groupe de Recherche 48 du CNRS. 
and extensed states for this model and obtained some results about the mobility edge. In particular we have proven that the density of states is analytic with respect to the energy and that the localization length $\xi$ diverges as $\xi(e) \sim\left(e-e_{\mathrm{c}}\right)^{-1}$ at the mobility edge, if the lengths are measured with the natural distance along the tree. At first sight, one might say that the critical exponent $v$ is equal to 1 ; however for comparison with the $d$-dimensional lattices, one has to reinterpret this result as $v=\frac{1}{2}$ for the same reasons that one has to do it in the case of the Ising model or for percolation [3]. On the other hand, Shapiro [4] has studied the quantum conductance $g$ on a Bethe lattice composed of one-dimensional scatterers of typical conductance $g_{0}$ and he obtains that the conductance should vanish at the transition as $g \sim\left(g_{0}-g_{0 c}\right)$, yielding an exponent $s=1$. His derivation is not rigorous, but is based on plausible approximations; we note however that Girvin and Johnson [5] found a jump in the conductivity on the Bethe lattice and hence an exponent $s=0$, but their work is only numerical and not free from affective approximations.

With our exact result for $v$ and if we admit the result of Shapiro for $s$, we have now two « classical " exponents (i.e. corresponding to the exponents of $d$-dimensional systems in the limit $d \rightarrow \infty$ ), namely $v=\frac{1}{2}$ and $s=1$. On the other hand, scaling theories for localization lead to the scaling relation $s=(d-2) v$ between these two exponents [6]; the validity of this relation is well established. The upper critical dimension $d_{c}$ should then be the one for which the scaling relation is satisfied by the " classical " value of the exponents. We are thus led to the conclusion $d_{\mathrm{c}}=4$ for the localization problem. This conclusion is at variance with suggestions or predictions of $d_{\mathrm{c}}=6, d_{\mathrm{c}}=8$ or $d_{\mathrm{c}}=\infty$.

More daring assumptions lead also to this conclusion. It has been proposed by Wegner [7] that the localization transition should be well described by non-linear $\sigma$ models at least if longitudinal modes can be neglected; these non-linear models are known to be renormalizable at least for dimensions $d=2$ and 3. But it was found in these models in a $d=2+\varepsilon$ expansion that the function $W(t)=\mathrm{d} t / \mathrm{d} \ln \mu$ describing the renormalization of the field theoretic coupling constant $t$ (which behaves as the inverse of the conductance) as a function of the momentum cut off $\mu$ (which behaves as the inverse of the length of the system) was given by $W(t)=\varepsilon t-$ $2 t^{2}+0\left(t^{5}\right)$. The vanishing of the $t^{3}$ and $t^{4}$ terms is due respectively to Brézin, Hikami and Zinn-Justin [8] and Hikami [9]. This expression of the function $W(t)$ leads to $v=\frac{1}{\varepsilon}+0\left(\varepsilon^{2}\right)$. It is then tempting, and this was already done by several persons, at least orally, to think, that the vanishing of both the third and the fourth order terms in the $W$ function is not an accident, and hence to question the possibility of a vanishing of all further corrections; if we make this hypothesis, we would get $v=1 /(d-2)$ up to the upper critical dimension where the expansion looses sense. Together with our exact result $v=\frac{1}{2}$, we again arrive at the prediction $d_{c}=4$. Alternatively, if one has already been convinced by the first argument of this note, we may turn the above remark into an argument in favour of the vanishing of all corrections in the $W$ function and, in fact, we conjecture it on this basis. We also note that the results on the $W$ function imply $s=1+0\left(\varepsilon^{3}\right)$; the hypothesis of the vanishing of corrections for $s$ does not lead to a prediction for the critical dimension but is consistent with the $s=1$ on the Bethe lattice that we used above.

We also remark that Wegner [7] has computed the behaviour of the inverse participation ratio $P(e)$ near $d=2$ and has found $P(e) \sim\left(e-e_{\mathrm{c}}\right)^{p}$ with $p=\frac{2-\varepsilon}{\varepsilon}+0(\varepsilon)$. If we accept as before that there are no corrections in $\varepsilon$ up to a critical dimension, we have $p=\frac{2-\varepsilon}{\varepsilon}$, which cannot hold for dimensions greater than 4. On the other hand, this would imply $p=0$ and $d_{\mathrm{c}}=4$, which is suggestive of a jump of the inverse participation ratio at the mobility edge; however a jump was obtained in an approximate treatment of the Bethe lattice model by Last and Thouless [10], the approximation giving a transition also for $d=1$, a situation quite reminiscent of a mean field theory. Here again, we have an argument suggesting for $d_{c}=4$. 
Let us finally mention the self-consistent theories developed following Götze [11], who was the first to have a theory describing both the localized and the extended regimes. Although, the first version of the theory did not obey the scaling relation of reference 6 and presented critical properties different from those obtained by $2+\varepsilon$ expansions, the incorporation of « quantum interferences " directly into the relaxation kernel [12] or a systematic analysis of diagrams [13] lead to an improved theory also satisfying the scaling relation and consistent with the $2+\varepsilon$ results except for the exponent $\eta$ which is 0 in self-consistent theories and $-\varepsilon$ in $2+\varepsilon$ theories. Although it is not clear that all other critical properties should be the same for the true Anderson model and for the self-consistent theories (the same reservation holds also for representation by non-linear $\sigma$-models) it is worth noting that in them the exponents $v$ and $s$ become constant for $d$ larger than 4, and lead then to $v=\frac{1}{2}$ as our result on the Bethe lattice.

Thus as we have seen there exist a number of arguments leading to $d_{c}=4$. In our mind the strongest is the first one based on the Bethe lattice results ; it is nice however to have other arguments converging to the same conclusion, although each of them is presently disputable. These arguments also lead to the conjecture that all corrections to the $W$ function should vanish exactly and make more desirable a computation of higher orders in the $(d-2)$-expansion of the exponents or an argument, perhaps based on supersymmetry ideas to imply systematic vanishing. (Indeed the vanishing of the terms appears only when the number of replicas goes to zero. It is possible to avoid the replica trick and formulate then the problem in terms of a supersymmetric non-linear $\sigma$-model [14], and we note that it is known that some supersymmetric field theories have « trivial " $W$ functions).

Another question of interest concerns what happens in the case when time-invariance is broken for which $d=2+\varepsilon$ expansions lead to $s=\frac{1}{2}+O(\varepsilon)$ and $v=\frac{1}{\varepsilon}+O(1)$. We have empirical arguments, although not yet a full mathematical proof that again on the Bethe lattice one should have $v=1$ which is to be reinterpreted as $v=\frac{1}{2}$. If we make again the assumption that all corrections vanish we obtain for this case $d_{c}=3$.

Finally we want to stress a consequence of our proposal. If we are right then some exponents are exactly known and in particular we should have $s=v=p=1$ for three-dimensional Anderson models.

\section{Acknowledgments.}

We are glad to thank E. Fradkin, R. Rammal, Y. Shapir, G. Toulouse and J. Vannimenus for a useful discussion.

\section{Note added in proof.}

Hikami (Nucl. Phys. B 215 (FS7) (1983) 555) has just published a calculation of the $t^{5}$ term of the $W(t)$ function of the non-linear $\sigma$-model. This term is also vanishing, and so this new result is consistent with our paper. We are pleased to thank the two referees of our paper for this information.

\section{References}

[1] Abou-Chacra, R., Anderson, P. W. and Thouless, D. J., J. Phys. C 6 (1973) 1734. Abou-Chacra, R. and Thouless, D. J., J. Phys. C 7 (1974) 65.

[2] Kunz, H. and Soulllard, B., The localization transition on the Bethe lattice. J. Physique Lett. 44 (1983).

[3] Vannimenus, J., Z. Phys. B 43 (1981) 141.

Straley, J. P., J. Phys. C 15 (1982) 2333.

[4] ShapIRo, B., Quantum conduction on a Cayley tree. Preprint. 
[5] Girvin, S. M. and Johnsón, M., Phys. Rev. B 22 (1980) 3583.

[6] Wegner, F., Z. Phys. B 25 (1976) 327.

Abrahams, E., Anderson, P. W., Licciardello, D. C. and Ramakrishnan, T. V., Phys. Rev. Lett. 42 (1979) 673.

[7] Wegner, F., Z. Phys. B 35 (1979) 207.

[8] Brezin, E., Hikami, S. and Zinn-Justin, J., Nucl. Phys. B 165 (1980) 528.

[9] Hikami, S., Phys. Rev. B 24 (1981) 2671.

[10] Last, B. J. and Thouless, D. J., J. Phys. C 7 (1974) 775.

[11] GöTZE, W., Solid State Commun. 27 (1978) 1393.

[12] Prelovšek, P., in Recent Developments in Condensed Matter Physcis, (Plenum Press) 1981.

Belitz, D., Gold, A., Götze, W., Z. Phys. B 44 (1981) 273.

[13] Vollhardt, D. and Wölfle, P., Phys. Rev. B 22 (1980) 4666.

Kawabata, A., Solid State Commun. 38 (1981) 823.

[14] Efetov, K. B., Sov. Phys. JETP 55 (1982) 3. 